\title{
Combined phacoemulsification, 23-gauge pars plana vitrectomy with internal limiting membrane peeling and gas tamponade for patients with coexisting idiopathic macular hole and age-associated cataract
}

\author{
QINGMIN MA, FANG FAN, ZHIHUA ZHAO and ZHIYANG JIA \\ Department of Ophthalmology, Hebei General Hospital, Shijiazhuang, Hebei 050051, P.R. China
}

Received April 28, 2018; Accepted September 19, 2018

DOI: $10.3892 /$ etm.2018.6963

\begin{abstract}
The aim of the present study was to assess the curative effect of combined phacoemulsification, 23-gauge pars plana vitrectomy with Brilliant blue G-assisted limiting membrane peeling and gas tamponade in patients with coexisting idiopathic macular hole (IMH) and age-associated cataract. A total of 21 consecutive patients (21 eyes) with coexisting IMH and age-associated cataract were enrolled in the study. All patients were treated by 23 -gauge microincision vitrectomy with internal limiting membrane peeling, gas tamponade and combined phacoemulsification. The pre-operative $\mathrm{MH}$ diameter, $\mathrm{MH}$ index and best-corrected visual acuity (BCVA), as well as events of post-operative $\mathrm{MH}$ closure and complications were recorded and analyzed. Anatomic closure of the MH was achieved in 19 eyes $(90.4 \%)$ with a single surgery. The LogMAR BCVA value at 1 month after surgery and the final follow-up visit was significantly lower than the baseline value $(\mathrm{P}=0.0036$ and $\mathrm{P}=0.0015$, respectively). A significant correlation was identified between the $\mathrm{MH}$ index and the post-operative LogMAR BCVA $(\mathrm{r}=0.869 ; \mathrm{P}<0.001)$. The combined technique applied in the present study produced favorable anatomic and functional results for patients with coexisting IMH and age-associated cataracts. The pre-operative $\mathrm{MH}$ size measured by optical coherence tomography may serve as a predictive factor for the LogMAR BCVA value following MH surgery.
\end{abstract}

\section{Introduction}

Idiopathic macular holes (IMH) are a major vitreoretinal pathology that may cause metamorphopsia and poor central

Correspondence to: Dr Zhiyang Jia, Department of Ophthalmology, Hebei General Hospital, 348 Heping West Road, Shijiazhuang, Hebei 050051, P.R. China

E-mail: jiazhiyang_med@163.com

Key words: pars plana vitrectomy, idiopathic macular hole, age-associated cataract, best-corrected visual acuity, 23-gauge vision $(1,2)$. IMH mainly occurs in individuals aged $60-70$ years, with $\sim 66.7 \%$ of those affected being female (3-6).

In the past, MHs were considered to be incurable. Vitrectomy in combination with intraocular gas tamponade was the first remedy for MHs with a prominent success rate $(7,8)$. Various adjunctive therapeutic strategies have also been introduced for MH treatment. Internal limiting membrane (ILM) peeling, an alternative treatment method, may increase the probability of primary anatomical closure of MHs (9-11). Previous studies have confirmed the safety and reliability of the 25-gauge vitrectomy system with sutureless self-sealing sclerotomies in the treatment of various vitreoretinal diseases $(12,13)$. The 23 -gauge transconjunctival system was then proposed, which combined the merits of 25- and 20-gauge instrumentation (14). In general, small-gauge transconjunctival vitrectomy has gained wide recognition due to its intra- and post-operative superiority, as it involves less pain, a shorter surgical time and a lower rate of post-operative inflammation (13).

Closed pars plana vitrectomy remains the primary treatment option for a large number of patients with IMH (15). Despite the great progress made in transconjunctival vitrectomy and the high post-operative anatomic success $(7,16-18)$, visual recovery is far from satisfactory among patients with MHs $(19,20)$. Cataract progression is the most common complication following $\mathrm{MH}$ surgery, often deeming a second surgery necessary within 2 years. As elderly patients commonly present with MHs, numerous eyes affected may have concomitant IMH and cataracts. ILM peeling may be a beneficial adjunctive procedure for $\mathrm{MH}$ surgery and has been reported to increase $\mathrm{MH}$ closure rates (15). However, it is a complex procedure due to the lens opacity of the eyes of patients with IMH accompanied by cataracts (21).

The aim of the present study was to assess the curative effect of combined phacoemulsification, 23-gauge pars plana vitrectomy with Brilliant blue G (BBG)-assisted ILM peeling and gas tamponade in patients with IMH and age-associated cataracts.

\section{Patients and methods}

Patients. A total of 21 consecutive patients with IMH accompanied by age-associated cataracts who presented at 
the Department of Ophthalmology, Hebei General Hospital (Shijiazhuang, China) from January to November 2016 were enrolled in the present study. All patients were subjected to 23-gauge microincision vitrectomy, air tamponade and phacoemulsification. The study was approved by the institutional review board of Hebei General Hospital (Shijiazhuang, China). Written informed consent was obtained from each patient prior to surgery and each patient consented for their information to be used for the purpose of research.

Prior to the surgery, all patients received ophthalmic examinations, which included best-corrected visual acuity (BCVA) assessment, slit-lamp biomicroscopic examination, intraocular pressure measurement and indirect ophthalmoscopic examinations. The diagnosis of IMH was made based on fundus examination and optical coherence tomography (OCT). The IMH grading system used was the Gass classification (22).

The inclusion criteria included stage 2-4 IMH accompanied by age-associated cataracts. The exclusion criteria were high myopia ( $>8 \mathrm{D})$, history of retinal diseases, diabetes and traumatic $\mathrm{MH}$, history of vitreoretinal surgery, $\mathrm{MH}$ stage 1, macular cysts or secondary MHs.

Surgical technique. All surgeries were performed by a single experienced vitreoretinal surgeon using a Stellaris PC system (Bausch \& Lomb Inc., Bridgewater, NJ, USA). Following superficial anesthesia with $3 \mathrm{ml}$ proxymetacaine (Alcon, Fort Worth, TX, USA) and retrobulbar anesthesia with a (1:1) mixture of $2 \%$ lidocaine and $0.75 \%$ bupivacaine $(3 \mathrm{ml})$, phacoemulsification surgery was performed via a $3.2-\mathrm{mm}$ sclera tunnel incision. An ophthalmic viscosurgical device was used to expand and stabilize the capsular bag, in order to facilitate the implantation of a foldable water-based acrylic intraocular lens into the capsular bag. A three-port 23-gauge vitrectomy was then performed. During the vitrectomy, a posterior vitreous detachment was performed and the remnant posterior vitreous cortex was removed. Peripheral vitreous detachment was then performed. Following visualization using BBG dye [ILM Blue ${ }^{\circledast}$; Dutch Ophthalmic Research Center (International) B.V., Zuidland, the Netherlands], the ILM was peeled off around the MH using a pair of ILM forceps. Fluid-air exchange and intraocular tamponade with sterilized air was applied at the end of the procedure. Following surgery, all patients were instructed to maintain a face-down position for 2-3 days at the hospital.

Post-operative treatment consisted of Tobramycin Dexamethasone eye drops (Alcon), Pranoprofen eye drops (SENJU Pharmaceutical Co., Ltd., Osaka, Japan) for anti-inflammatory therapy and Tropicamide eye drops (Shenyang Xingqi Pharmaceutical Co., Ltd., Shengyang, China) for mydriasis treatment.

Clinical evaluation. The recorded data included the pre-operative $\mathrm{MH}$ diameter, the $\mathrm{MH}$ index (MHI), the LogMAR BCVA, as well as events of post-operative MH closure and any potential intra- and post-operative complications. The MH diameter, MHI and BCVA were measured at 1 month post-surgery and at the last follow-up. MH closure was considered an anatomical success when no neurosensory defects were observed in the fovea on ophthalmoscopy with
Table I. Baseline characteristics.

\begin{tabular}{lc}
\hline Item & Value \\
\hline Sex (male/female) & $5 / 16$ \\
Age (years) & $64.2 \pm 5.3$ \\
Eye (left/right) & $13 / 8$ \\
Hole diameter $(\mu \mathrm{m})$ & $504.0 \pm 212.7$ \\
MHI & $1.10 \pm 1.13$ \\
LogMAR BCVA & \\
Pre-operative & $1.22 \pm 0.42$ \\
Last follow-up & $0.75 \pm 0.47$ \\
Follow-up time (months) & $8.28 \pm 3.52$ \\
Post-operative complications (hemorrhage) & $2(9.5)$ \\
Cataract grade regarding lens nuclear sclerosis & \\
II & $8(38.1)$ \\
III & $12(57.1)$ \\
IV & $1(4.8)$ \\
\hline
\end{tabular}

Values are expressed as $\mathrm{n}(\%)$ or the mean \pm standard deviation. BCVA, best-corrected visual acuity; MHI, macular hole index.

Table II. Correlation between the closure of the $\mathrm{MH}$ and the MH diameter or the MH index at 1 month post-operation.

\begin{tabular}{lcc}
\hline \multicolumn{1}{l}{ Item } & $\begin{array}{c}\text { Post-operative } \\
\text { closure rate }(\%)\end{array}$ & P-value \\
\hline Pre-operative MH diameter $(\mu \mathrm{m})$ & & \\
$<300(\mathrm{n}=6)$ & 100 & \\
$300-600(\mathrm{n}=7)$ & 100 & $>0.999^{\mathrm{a}}$ \\
$>600(\mathrm{n}=8)$ & 75 & $0.13^{\mathrm{b}}$ \\
Pre-operative MH index & & \\
$<0.5(\mathrm{n}=10)$ & 90 & 0.48 \\
$\geq 0.5(\mathrm{n}=11)$ & 100 & \\
\hline
\end{tabular}

${ }^{\mathrm{a} C}$ Closure rate in patients with a $\mathrm{MH}$ diameter $<300$ vs. $\geq 300 \mu \mathrm{m}$, ${ }^{\mathrm{b}} \mathrm{Closure}$ rate in patients with a $\mathrm{MH}$ diameter $>600 \mathrm{vs} . \leq 600 \mu \mathrm{m}$. MH closure, macular hole closure at last follow-up. $\mathrm{MH}$, macular hole.

confirmation by OCT (23). Post-operative complications were observed by slit-lamp biomicroscopy, indirect funduscopy and intraocular pressure assessment.

Statistical analysis. Quantitative data are expressed as the mean \pm standard deviation and analyzed using repeated-measures analysis of variance followed by the Bonferroni test. Qualitative data are presented as numbers and/or percentages and were analyzed using the $\chi^{2}$ or Fisher's exact test, as appropriate. Correlation between the pre-operative $\mathrm{MH}$ diameter and the post-operative LogMAR BCVA was analyzed using a Spearman rank correlation analysis. Statistical analysis was performed using SPSS 19.0 software (IBM Corp., Armonk, NY, USA). $\mathrm{P}<0.05$ was considered to indicate a statistically significant difference. 

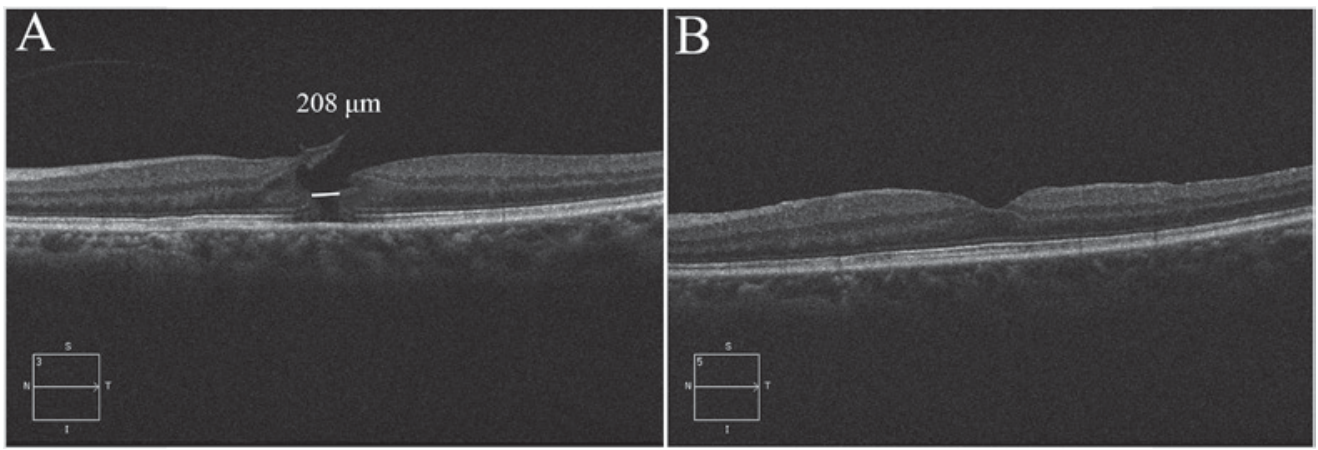

Figure 1. Pre- and post-operative OCT images of a 66-year-old female who presented with dysmorphopsia for 1 month. (A) Pre-operative OCT image of the MH, with an MHI of 4.221 and a hole diameter of $208 \mu \mathrm{m}$. (B) Post-operative OCT image displaying anatomical closure of the MH at 12 months after surgery. OCT, optical coherence tomography; MH, macular hole; MHI, MH index.

\section{Results}

Baseline characteristics. A total of 21 patients, including 5 men $(23.8 \%)$ and 16 women $(76.2 \%)$ and 21 eyes, diagnosed with coexisting IMH and age-associated cataracts were enrolled in the present study. The mean age of the patients was $64.2 \pm 5.3$ years (range, 51-72 years). The demographic data of the patients are listed in Table I. The mean post-operative follow-up duration was 8.28 \pm 3.52 months (range, 1-12 months) and the mean value of the pre-operative $\mathrm{MH}$ diameter was $504.0 \pm 212.7 \mu \mathrm{m}$. Of the 21 cases, 8 patients $(38.1 \%)$ presented with grade II and $12(57.1 \%)$ with grade III nuclear sclerosis.

Initial hole-closure rate. Anatomic $\mathrm{MH}$ closure was achieved in 19 eyes $(90.4 \%$ ) with a single surgery (Fig. 1), while 2 eyes did not achieve $\mathrm{MH}$ closure following initial surgery. These latter 2 patients were asked to maintain a face-down position for 2 weeks after the MHs were sealed using autologous serum and silicone oil tamponades. Anatomical closure of the MHs and improvement in VA was observed in these 2 cases at 1 month after surgery. A higher $\mathrm{MH}$ closure rate was observed in patients with an $\mathrm{MH}$ diameter of $\leq 600 \mu \mathrm{m}$, as compared with that in patients with an $\mathrm{MH}$ diameter of $>600 \mu \mathrm{m}$; however, the difference was not significant $(\mathrm{P}=0.13)$. No significant association was observed between $\mathrm{MH}$ index and closure rate ( $\mathrm{P}=0.48$; Table II).

$B C V A$. The mean pre-operative LogMAR BCVA was $1.22 \pm 0.42$. Following combined surgery, the mean LogMAR BCVA decreased to $0.82 \pm 0.43$ and $0.75 \pm 0.47$, at 1 month post-surgery and the last follow-up, respectively. A significant decrease in the LogMAR BCVA was observed at 1 month post-surgery $(\mathrm{P}=0.0036)$ and at the last follow-up $(\mathrm{P}=0.0015)$, as compared with the baseline levels (Fig. 2A). Spearman rank correlation analysis revealed a positive correlation between the pre-operative $\mathrm{MH}$ diameter and the post-operative LogMAR BCVA ( $r=0.869, \mathrm{P}<0.001$; Fig. 2B).

Intraocular pressure. The intraocular pressure prior to surgery, at post-operative month 1 and the last follow-up was $12.3 \pm 2.2,14.8 \pm 3.7$ and $11.4 \pm 2.8 \mathrm{mmHg}$, respectively. No statistically significant difference in intraocular pressure from baseline was observed at 1 month post-surgery and at the final follow-up (all P>0.05; Fig. 2C).
Complications. Post-operative vitreous hemorrhage was observed in 2 eyes and they were successfully treated by vitreous lavage. No choroidal detachment or endophthalmitis was recorded.

\section{Discussion}

IMHs are full-thickness defects of the retinal tissue involving the anatomic fovea. They may affect central visual acuity and even cause metamorphopsia. As MH commonly occurs in aged patients, numerous eyes affected may have concomitant $\mathrm{MH}$ and cataract. Despite the great progress in transconjunctival vitrectomy and high anatomic success after surgery, visual recovery in $\mathrm{MH}$ patients is far from satisfactory. Therefore, a more efficient surgical method is necessary. The success rate of $\mathrm{MH}$ surgery has been increased to $90 \%$ since $\mathrm{MH}$ repair was first described (24). However, few studies have assessed combined phacoemulsifcation with 23-gauge pars plana vitrectomy with BBG-assisted ILM peeling and gas tamponade $(13,25,26)$. The present study combined cataract and $\mathrm{MH}$ surgery, including phacoemulsifcation, 23-gauge pars plana vitrectomy, BBG-assisted ILM peeling and gas tamponade. All patients received phacoemulsification, pars plana vitrectomy, ILM peeling and fluid-gas exchange, and a face-down position was adopted for several days after the surgery. The $\mathrm{MH}$ closure rate of $90.4 \%$ determined in the present study was consistent with the success rates reported in previous studies (27-29). Rizzo et al (27) revealed that $\mathrm{MH}$ closure rate was $93 \%$ following the use of a sutureless 25 -gauge vitrectomy (27). Furthermore, the macular hole closure rate was $\sim 90 \%$ with sulfur hexafluoride gas or perfluoropropane gas (28). A comparatively higher $\mathrm{MH}$ closure rate was observed in patients with a hole diameter of $\leq 600 \mu \mathrm{m}$, but no significant difference was observed. These results were inconsistent with those of previous studies reporting that a smaller $\mathrm{MH}$ is associated with a better prognosis regarding post-operative anatomical closure (30-32). This contradiction may be due to the fact that the sample size of the present study was too small to reach statistical significance. Therefore, further studies with a larger sample size are required to clarify this.

The effect of the pre-operative MHI on post-operative anatomical closure was also determined. The present data indicated that the MHI had no significant predictive value. By contrast, Kusuhara et al (33) reported that an MHI of $\geq 0.5$ is 
A
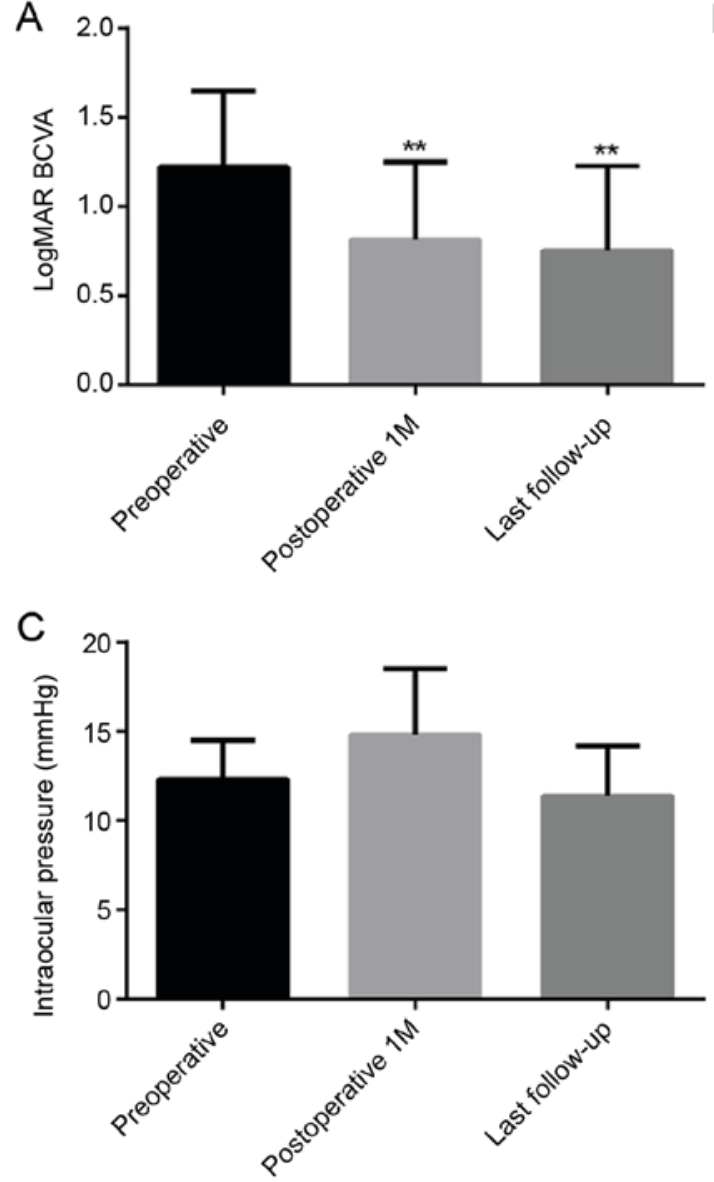

B

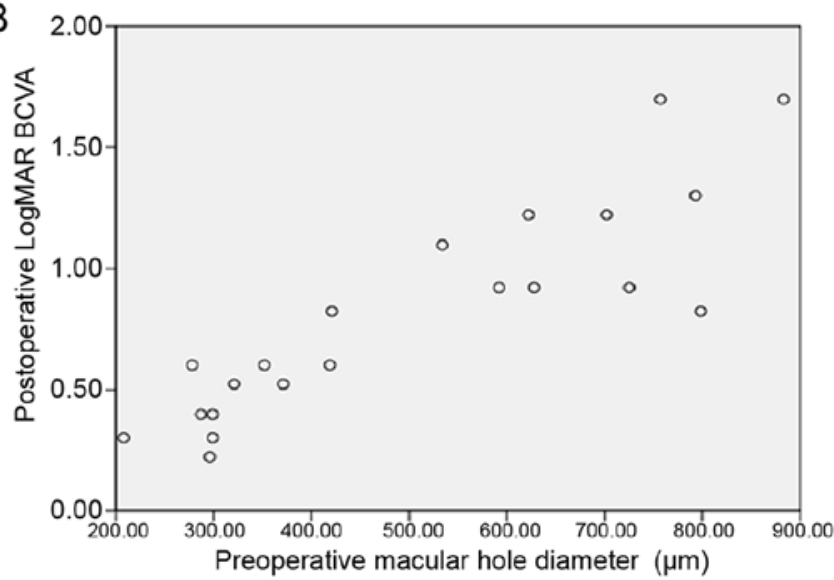

Figure 2. (A) LogMAR BCVA value at the pre-operative stage, at 1 month post-surgery and at the last follow-up. ${ }^{* *} \mathrm{P}<0.01$ vs. pre-operative. (B) Spearman rank analysis revealed a positive correlation between the pre-operative macular hole diameter and the post-operative LogMAR BCVA ( $\mathrm{P}<0.001, \mathrm{r}=0.869)$. (C) Intraocular pressure at the pre-operative stage, at 1month post-surgery and at the last follow-up. BCVA, best-corrected visual acuity; OCT, optical coherence tomography.

a positive predictive factor for a favorable surgical outcome. This contradiction may be due to the fact that the present study included a small number of cases and that large inter-individual differences were present. Furthermore, the results indicated a positive correlation between the pre-operative $\mathrm{MH}$ diameter and the post-operative LogMAR BCVA. This suggests that the pre-operative $\mathrm{MH}$ size measured by OCT may provide a predictive factor for the LogMAR BCVA after MH surgery. Therefore, apart from proposing another effective combined surgical method, the present study may also provide a potential predictor for the treatment outcome of MH. The 23-gauge vitrectomy system allows for a small incision, self-sealing and sutureless transconjunctival pars plana sclerotomies, which renders it superior to the traditional 20 -gauge vitrectomy system (34). In the present study, 2 eyes presented with post-operative vitreous hemorrhage, which was thought to be associated with sutureless transconjunctival pars plana sclerotomies and low intraocular pressure in the affected patients at the end of the air tamponade.

MHs often occur in aged/elderly individuals, and thus, certain patients may present with co-existing $\mathrm{MH}$ and cataract. Due to blurred media, vitrectomy surgery becomes more difficult. Combined cataract extraction and vitrectomy has been regarded as an effective method for the treatment of various vitreoretinal diseases $(21,25,26,35-37)$.
Muselier et al (21) reported that combined and consecutive surgeries produced equivalent functional and anatomical results. Combined cataract and MH surgery has been reported to be more advantageous than consecutive surgeries, as it avoids a second surgical procedure and is more convenient for the vitreoretinal surgeon (38). In addition, in this study, a faster visual recovery was observed following the combined surgery; thus, a second surgery was avoided and the overall surgical cost was reduced. A previous study by Hikichi et al (13) has confirmed the effectiveness of 23-gauge vitrectomy with air tamponade and combined phacoemulsification, but without the BBG-assisted ILM peeling, for IMH. However, the cases included were of IMH with no cataracts. The present study assessed the combined surgery in patients with coexisting IMH and age-associated cataracts. Demetriades et al (26) have reported that combined phacoemulsification, intraocular lens implantation and vitrectomy is a reasonable alternative in patients with coexisting cataract and vitreoretinal pathology. However, the combined surgery was not applied for the treatment of MH. Thus, the present study provided a novel effective combined surgery for the treatment of patients with coexisting MHs and cataracts.

Combining surgeries has numerous advantages (21,38-41). First, patients do not require a second surgical procedure. Furthermore, it is beneficial for extending vitrectomy, which 
contributes to the removal of the anterior vitreous. Increasing the gas volume may provide a longer tamponade, which may prove beneficial for the closure of the MH. Nuclear sclerotic cataract progression has been associated with the use of vitrectomy for the treatment of MHs (42). Therefore, combined cataract extraction and vitrectomy may help avoid the adverse effects of cataract progression despite improvement in visual acuity. Muselier et al (21) reported a marked difference in visual acuity improvement at 0.5 years after surgery between patients who underwent vitrectomy alone and those who underwent vitrectomy combined with cataract extraction for the treatment of MHs. The study concluded that cataract progression following vitreous surgery may have been due to the slower recovery of patients who received a separate vitrectomy (21).

The present study had certain limitations. First, it lacks a concurrent control group. Furthermore, all procedures were performed by a single surgeon, thereby failing to compare the differences in clinical outcomes between different surgical procedures or surgeons. Finally, the size of the study population was small due to the strict inclusion and exclusion criteria. However, the present results may be representative for a patient population similar to that of the present study, namely those with coexisting IMH and age-associated cataract.

In conclusion, combined phacoemulsification with 23-gauge pars plana vitrectomy with BBG-assisted ILM peeling and gas tamponade yielded satisfactory anatomic and functional results for patients with IMH and age-associated cataracts. The pre-operative MH size measured by OCT may be a predictive factor for the LogMAR BCVA after $\mathrm{MH}$ surgery.

\section{Acknowledgements}

Not applicable.

\section{Funding}

No funding was received.

\section{Availability of data and materials}

The datasets used and/or analyzed during the present study are available from the corresponding author on reasonable request.

\section{Authors' contributions}

QM drafted the manuscript. ZJ designed the study. QM and FF analyzed and interpreted the data. ZZ collected and assembled the data. All authors read and approved the final manuscript.

\section{Ethics approval and consent to participate}

This study was approved by the ethics committee of Hebei General Hospital. All patients provided written informed consent.

\section{Patient sconsent for publication}

All patients have provided informed consent for publication.

\section{Competing interests}

The authors declare that they have no competing interests.

\section{References}

1. Wang S, Xu L and Jonas JB: Prevalence of full-thickness macular holes in urban and rural adult Chinese: The Beijing Eye Study. Am J Ophthalmol 141: 589-591, 2006.

2. Jackson TL, Donachie PH, Sparrow JM and Johnston RL: United Kingdom National Ophthalmology Database study of vitreoretinal surgery: Report 2, macular hole. Ophthalmology 120: 629-634, 2013.

3. Casuso LA, Scott IU, Flynn HW Jr, Gass JD, Smiddy WE, Lewis ML and Schiffman J: Long-term follow-up of unoperated macular holes. Ophthalmology 108: 1150-1155, 2001.

4. Kim JW, Freeman WR, el-Haig W, Maguire AM, Arevalo JF and Azen SP: Baseline characteristics, natural history, and risk factors to progression in eyes with stage 2 macular holes. Results from a prospective randomized clinical trial. Vitrectomy for Macular Hole Study Group. Ophthalmology 102: 1818-1828; discussion 1828-1819, 1995.

5. Jackson TL, Donachie PH, Sparrow JM and Johnston RL: United Kingdom National Ophthalmology Database study of vitreoretinal surgery: Report 1; case mix, complications, and cataract. Eye (Lond) 27: 644-651, 2013.

6. Risk factors for idiopathic macular holes. The Eye Disease Case-Control Study Group. Am J Ophthalmol 118: 754-761, 1994.

7. Kelly NE and Wendel RT: Vitreous surgery for idiopathic macular holes. Results of a pilot study. Arch Ophthalmol 109: 654-659, 1991.

8. Wendel RT, Patel AC, Kelly NE, Salzano TC, Wells JW and Novack GD: Vitreous surgery for macular holes. Ophthalmology 100: 1671-1676, 1993.

9. Tognetto D, Grandin R, Sanguinetti G, Minutola D, Di Nicola M, Di Mascio R and Ravalico G; Macular Hole Surgery Study G: Internal limiting membrane removal during macular hole surgery: Results of a multicenter retrospective study. Ophthalmology 113: 1401-1410, 2006.

10. Sheidow TG, Blinder KJ, Holekamp N, Joseph D, Shah G, Grand MG, Thomas MA, Bakal J and Sharma S: Outcome results in macular hole surgery: An evaluation of internal limiting membrane peeling with and without indocyanine green. Ophthalmology 110: 1697-1701, 2003.

11. Lois N, Burr J, Norrie J, Vale L, Cook J, McDonald A, Boachie C, Ternent L and McPherson G; Full-thickness Macular Hole and Internal LimitingMembrane Peeling Study (FILMS) Group: Internal limiting membrane peeling versus no peeling for idiopathic full-thickness macular hole: A pragmatic randomized controlled trial. Invest Ophthalmol Vis Sci 52: 1586-1592, 2011.

12. Rizzo S, Genovesi-Ebert F, Murri S, Belting C, Vento A, Cresti F and Manca ML: 25-gauge, sutureless vitrectomy and standard 20-gauge pars plana vitrectomy in idiopathic epiretinal membrane surgery: A comparative pilot study. Graefes Arch Clin Exp Ophthalmol 244: 472-479, 2006.

13. Hikichi T, Kosaka S, Takami K, Ariga H, Ohtsuka H, Higuchi M, Matsushita T and Matsushita R: 23- and 20-gauge vitrectomy with air tamponade with combined phacoemulsification for idiopathic macular hole: A single-surgeon study. Am J Ophthalmol 152: 114-121 e1, 2011.

14. Goncu T, Gurelik G and Hasanreisoglu B: Comparison of efficacy and safety between transconjunctival 23-gauge and conventional 20 -gauge vitrectomy systems in macular surgery. Korean J Ophthalmol 26: 339-346, 2012.

15. Steel DH and Lotery AJ: Idiopathic vitreomacular traction and macular hole: A comprehensive review of pathophysiology, diagnosis, and treatment. Eye (Lond) 27 (Suppl 1): S1-S21, 2013.

16. Wittich W, Overbury O, Kapusta MA and Faubert J: Visual function assessment and metamorphopsia after macular hole surgery. Ophthalmic Physiol Opt 25: 534-542, 2005.

17. Grigoropoulos VG, Theodossiadis GP and Theodossiadis PG: Association of the preoperative photoreceptor layer defect as assessed by optical coherence tomography with the functional outcome after macular hole closure: A long follow-up study. Ophthalmologica 225: 47-54, 2011. 
18. Passemard M, Yakoubi Y, Muselier A, Hubert I, Guillaubey A Bron AM, Berrod JP and Creuzot-Garcher C: Long-term outcome of idiopathic macular hole surgery. Am J Ophthalmol 149: $120-126,2010$

19. Haritoglou C, Gass CA, Schaumberger M, Ehrt O, Gandorfer A and Kampik A: Macular changes after peeling of the internal limiting membrane in macular hole surgery. Am J Ophthalmol 132: 363-368, 2001.

20. Tranos PG, Ghazi-Nouri SM, Rubin GS, Adams ZC and Charteris DG: Visual function and subjective perception of visual ability after macular hole surgery. Am J Ophthalmol 138: 995-1002, 2004

21. Muselier A, Dugas B, Burelle X, Passemard M, Hubert I, Mathieu B, Berrod JP, Bron AM and Creuzot-Garcher C: Macular hole surgery and cataract extraction: Combined vs consecutive surgery. Am J Ophthalmol 150: 387-391, 2010.

22. Gass JD: Reappraisal of biomicroscopic classification of stages of development of a macular hole. Am J Ophthalmol 119: 752-759, 1995.

23. Abdelkader MF and Moharram HM: Internal limiting membrane closure of idiopathic macular hole. J Clin Exp Ophthalmol 7: 618, 2016.

24. Wu D, Ho LY, Lai M and Capone A Jr: Surgical outcomes of idiopathic macular hole repair with limited postoperative positioning. Retina 31: 609-611, 2011.

25. Koenig SB, Mieler WF, Han DP and Abrams GW: Combined phacoemulsification, pars plana vitrectomy, and posterior chamber intraocular lens insertion. Arch Ophthalmol 110 1101-1104, 1992.

26. Demetriades AM, Gottsch JD, Thomsen R, Azab A, Stark WJ Campochiaro PA, de Juan E Jr and Haller JA: Combined phacoemulsification, intraocular lens implantation, and vitrectomy for eyes with coexisting cataract and vitreoretinal pathology. Am J Ophthalmol 135: 291-296, 2003.

27. Rizzo S, Belting C, Cresti F and Genovesi-Ebert F: Sutureless 25 -gauge vitrectomy for idiopathic macular hole repair. Graefes Arch Clin Exp Ophthalmol 245: 1437-1440, 2007.

28. Kim SS, Smiddy WE, Feuer WJ and Shi W: Outcomes of sulfur hexafluoride (SF6) versus perfluoropropane (C3F8) gas tamponade for macular hole surgery. Retina 28: 1408-1415, 2008.

29. Lahey JM, Francis RR, Fong DS, Kearney JJ and Tanaka S: Combining phacoemulsification with vitrectomy for treatment of macular holes. Br J Ophthalmol 86: 876-878, 2002.

30. Haritoglou C, Neubauer AS, Reiniger IW, Priglinger SG, Gass CA and Kampik A: Long-term functional outcome of macular hole surgery correlated to optical coherence tomography measurements. Clin Exp Ophthalmol 35: 208-213, 2007.

31. Yamamoto $\mathrm{K}$ and Hori S: Long-term outcome of vitrectomy combined with internal limiting membrane peeling for idiopathic macular holes. Nippon Ganka Gakkai Zasshi 115: 20-26, 2011 (In Japanese).
32. Ullrich S, Haritoglou C, Gass C, Schaumberger M, Ulbig MW and Kampik A: Macular hole size as a prognostic factor in macular hole surgery. Br J Ophthalmol 86: 390-393, 2002.

33. Kusuhara S, Teraoka Escaño MF, Fujii S, Nakanishi Y, Tamura Y, Nagai A, Yamamoto H, Tsukahara Y and Negi A: Prediction of postoperative visual outcome based on hole configuration by optical coherence tomography in eyes with idiopathic macular holes. Am J Ophthalmol 138: 709-716, 2004.

34. Misra A, Ho-Yen G and Burton RL: 23-gauge sutureless vitrectomy and 20-gauge vitrectomy: A case series comparison. Eye (Lond) 23: 1187-1191, 2009.

35. Androudi S, Ahmed M, Fiore T, Brazitikos P and Foster CS: Combined pars plana vitrectomy and phacoemulsification to restore visual acuity in patients with chronic uveitis. J Cataract Refract Surg 31: 472-478, 2005.

36. Chung TY, Chung $\mathrm{H}$ and Lee JH: Combined surgery and sequential surgery comprising phacoemulsification, pars plana vitrectomy, and intraocular lens implantation: Comparison of clinical outcomes. J Cataract Refract Surg 28: 2001-2005, 2002.

37. Dugas B, Ouled-Moussa R, Lafontaine PO, Guillaubey A, Berrod JP, Hubert I, Bron AM and Creuzot-Garcher CP: Idiopathic epiretinal macular membrane and cataract extraction: Combined versus consecutive surgery. Am J Ophthalmol 149: 302-306, 2010.

38. Treumer F, Bunse A, Rudolf M and Roider J: Pars plana vitrectomy, phacoemulsification and intraocular lens implantation. Comparison of clinical complications in a combined versus two-step surgical approach. Graefes Arch Clin Exp Ophthalmol 244: 808-815, 2006.

39. Satchi K and Patel CK: Posterior chamber compartments demonstrated by optical coherence tomography, in silicone-filled eyes, following macular hole surgery. Clin Exp Ophthalmol 33: 619-622, 2005.

40. Grusha YO, Masket S and Miller KM: Phacoemulsification and lens implantation after pars plana vitrectomy. Ophthalmology 105: 287-294, 1998.

41. Creuzot-Garcher C, Aubé H, Candé F, Dupont G, Guillaubey A, Malvitte L, Arnavielle S and Bron A: Vitreoretinal outpatient surgery: Clinical and financial considerations. J Fr Ophtalmol 31: $871-876,2008$.

42. Thompson JT, Glaser BM, Sjaarda RN and Murphy RP: Progression of nuclear sclerosis and long-term visual results of vitrectomy with transforming growth factor beta-2 for macular holes. Am J Ophthalmol 119: 48-54, 1995. 